Technical note

\title{
Implementing safe and robust Total Marrow Irradiation using Helical Tomotherapy - A practical guide
}

\author{
André Haraldsson $^{\mathrm{a}, \mathrm{b}, *}$, Jacob Engellau ${ }^{\mathrm{a}, \mathrm{b}}$, Stig Lenhoff ${ }^{\mathrm{b}}$, Silke Engelholm ${ }^{\mathrm{b}}$, Sven Bäck ${ }^{\mathrm{b}}$,
} Per E. Engström ${ }^{\mathrm{b}}$

${ }^{a}$ Medical Radiation Physics, Lund University, Lund, Sweden

${ }^{\mathrm{b}}$ Department of Haematology, Oncology and Radiation Physics, Skåne University Hospital, Lund, Sweden

\section{A R T I C L E I N F O}

\section{Keywords:}

TMI

Total

Marrow

Irradiation

Radiotherapy

Tomotherapy

Helical

\begin{abstract}
A B S T R A C T
Total Marrow Irradiation (TMI) with Helical Tomotherapy is a radiotherapy treatment technique that targets bone marrow and sanctuary sites prior to stem cell or bone marrow transplantation (SCT/BMT). TMI is a complex procedure that involves several critical steps that all need to be carefully addressed for a successful implementation, such as dose homogeneity in field junctions, choice of target margins, integrity of treatment and back-up planning. In this work we present our solution for a robust and reproducible workflow throughout the treatment chain and data for twenty-three patients treated to date.

Material \& Methods: Patients were immobilized in a whole body vacuum cushion and thermoplastic mask. CTscanning and treatment were performed in two parts with field matching at the upper thigh. Target consisted of marrow containing bone and sanctuary sites. Lungs, kidneys, bowel, heart and liver were defined as organs at risk (OAR). A fast surface scanning system was used to position parts of the body not covered by the imaging system (MVCT) as well as to reduce treatment time.

Results: All patients completed their treatment and could proceed with SCT/BMT. Doses to OARs were significantly reduced and target dose homogeneity was improved compared to TBI. Robustness tests performed on field matching and patient positioning support that the field junction technique is adequate. Replacing MVCT with optical surface scanning reduced the treatment time by $25 \mathrm{~min}$ per fraction.

Conclusion: The methodology presented here has shown to provide a safe, robust and reproducible treatment for Total Marrow Irradiation using Tomotherapy.
\end{abstract}

\section{Introduction}

Total body irradiation (TBI) is used in conditioning regimes to suppress the immune system and eradicate tumor cells prior to hematopoietic stem cell transplantation (SCT). The treatment's viability is shown by the lower relapse rate when patients are treated with radiotherapy and chemotherapy versus chemotherapy alone $[1,2]$.

The standard treatment for TBI is anterior-posterior irradiation using a conventional linear accelerator at extended source-to-skin distance (SSD), with lead blocks shielding the lungs. This technique is simple, robust, and can be implemented on virtually any linear accelerator provided the treatment room is sufficiently large. However, the treatment often results in large dose heterogeneities in the patient, and the technique offers no sparing of organs at risk (OAR) except for lungs. The treatment is toxic, especially in combination with chemotherapy [3] and may cause significant side effects to a number of organs such as lungs, kidneys, bowel, and liver. The high toxicity has been shown to rule out attempted dose escalation, and lower relapse rate with higher dose has not affected the overall survival [4]. Helical Tomotherapy (HT) is a treatment modality that combines continuous gantry rotation and couch translation during irradiation. This allows treating targets up to about $135 \mathrm{~cm}$ length without any field junctions. These properties make HT a powerful option for irradiating long complex targets such as bone marrow $[5,6]$, although TMI treatments need to be split in two fields due to treatment length exceeding the $135 \mathrm{~cm}$ limit. Total Marrow Irradiation using Helical Tomotherapy to target bone marrow has been described previously [5,7-14]. However, the aim of this work is to present a straightforward guideline for implementing safe and robust Total Marrow Irradiation in a single Tomotherapy unit clinic. We believe that there is great gain in TMI treatment for these patients but challenges in implementing such a complex technique need to be addressed and illustrated. Here we describe a working procedure for

\footnotetext{
* Corresponding author at: Skånes Universitetssjukhus, Klinikgatan 5, 22185 Lund, Sweden.

E-mail address: andre.haraldsson@med.lu.se (A. Haraldsson).
} 
immobilization, beam junction optimization, surface scanning for patient positioning and backup planning for TMI. Potential pitfalls, difficulties and solutions are discussed for the entire treatment chain.

\section{Material \& Methods}

A multidisciplinary group consisting of all personnel involved in the TMI treatment was formed to develop and implement the treatment process. The TMI treatment chain included: determination of the conditioning regimen for radiotherapy, patient immobilization, computed tomography (CT), target and OAR delineation, treatment planning, quality control (QC), setup and imaging, treatment and backup planning.

\subsection{Patient characteristics}

Twenty-three patients presented with a wide range of body mass indices, ranging from 15 to 40, of which 7 (41\%) were females. The median age of the patients was 22 years, range $12-58$ and 4 out of 17 (24\%) were paediatric patients aged $12,12,14$ and 18 years. All patients were heavily pre-treated, and all but one were referred to the department of radiotherapy from the department of haematology. One paediatric patient was referred from another hospital, and treated with a reversed order of conditioning - TMI prior to chemotherapy. Standard radiotherapy prescription was $12 \mathrm{~Gy}$ given in 6 fractions twice daily over 3 days, with minimum $6 \mathrm{~h}$ between fractions. The characteristics, diagnosis and fractionation regimes of the patients are presented in Table 1. All patients were informed of the TMI treatment and the conventional TBI treatment, and all consented to undergo TMI treatment. The study has been approved by the local ethics committee.

\subsection{Immobilization}

Prior to CT acquisition, patients were firmly and reproducibly immobilized in a large vacuum cushion (VacFix, Par Scientific A/S, Odense Denmark) encompassing the entire body (Fig. 1). A 5 point open-face thermoplastic mask (Orfit Industries, Wijnegem, Belgium) together with an individually moulded neck support (MOLDCARE, Qfix,
PA, US) was used to immobilize the head and shoulders. Cotton pads served as spacers between mask and body to enable room for port-acath and central venous catheter. Hands and arms were placed as tight as possible alongside the body since minimising the distance between the arms improves the target dose homogeneity [15] and also maximises the body volume within the field of view of the MVCT.

Knees were slightly flexed to minimise the curvature of the lumbar vertebrae which we found facilitated the setup reproducibility. Feet were immobilized with firm imprints of the heels.

\subsection{Computer tomography}

All patients were CT-scanned in two parts of opposite direction due to the limited treatment length of the Tomotherapy unit $(135 \mathrm{~cm})$. First, from vertex to thigh in head first supine position (HFS) followed by the second scan from toes to upper thigh in feet-first supine position (FFS) using the same immobilization but rotated 180 degrees and with net immobilization removed. The two scans, acquired using $5 \mathrm{~mm}$ slice thickness, overlapped by approximately $20 \mathrm{~cm}$ to allow sufficient image data for accurate registration of the two CT-sets later in the dose summation phase (Fig. 2). Multiple transversal lines were drawn on all extremities together with corresponding positions on the vacuum cushion (Fig. 1). Two internal reference points were defined with tattoos and fiducial markers; for the upper body at the level of mammillae and for the lower body (legs) at the level of the field junction on the upper thighs. The fiducial junction markers on the thigh were encompassed by both CT-sets. To avoid image acquisition in deep inspiration or expiration phase, the patient was instructed to breathe calmly to ensure a CT scan representative of the treatment position.

\subsection{Structure delineation}

Following CT, both scans were exported to the treatment planning system (TPS) Eclipse (Varian Medical Systems) where OARs and target structures were delineated. All relevant OARs were delineated for dose monitoring purposes but only heart, bowel, liver, lungs and kidneys were used in the optimisation process.

Clinical target volume (CTV) was defined as bone and auto-

Table 1

Patients treated with Total Marrow Irradiation 2014-2018. Basic patient information is presented with diagnosis and treatment given as well as targeted organs. Patient \#6 received an additional boost to $20 \mathrm{~Gy} / 5 \mathrm{~F}$ to the right humerus, and thoracic columna due to FDG-PET positive myeloma involvement and patient \#10 received a boost to enlarged lymph nodes. Deviations from standard prescription (12 Gy) by request from referring haematologist.

\begin{tabular}{|c|c|c|c|c|c|}
\hline Patient & Sex/Age (years) & BMI & Diagnosis & Fractionation & Target volumes \\
\hline 1 & Female (49) & 40 & 8p11 MPD + T-ALL & $12 \mathrm{~Gy} / 6 \mathrm{~F}$ b.i.d & BM, CNS, Spleen \\
\hline 2 & Male (45) & 29 & CML + ALL recurrence & $12 \mathrm{~Gy} / 6 \mathrm{~F}$ b.i.d & BM, CNS, Spleen, scrotum \\
\hline 3 & Female (14) & 29 & Pre-B ALL recurrence & $12 \mathrm{~Gy} / 6 \mathrm{~F}$ b.i.d & BM, CNS, Spleen \\
\hline 4 & Male (22) & 24 & $\mathrm{Ph}+$ pre-B ALL & $12 \mathrm{~Gy} / 6 \mathrm{~F}$ b.i.d & BM, CNS, Spleen, Scrotum \\
\hline 5 & Female (12) & 15 & Pre-B ALL recurrence & $12 \mathrm{~Gy} / 6 \mathrm{~F}$ b.i.d & BM, CNS, Spleen \\
\hline 6 & Male (18) & 25 & Myeloma & $12 \mathrm{~Gy} / 6 \mathrm{~F}$ b.i.d & $\mathrm{BM}$ \\
\hline 7 & Male (22) & 24 & Pre-B ALL & $12 \mathrm{~Gy} / 6 \mathrm{~F}$ b.i.d & BM, CNS, Spleen, Scrotum \\
\hline 8 & Male (40) & 24 & $\mathrm{Ph}+$ Pre-B ALL & 8 Gy/4F b.i.d & BM, CNS, Spleen, Scrotum \\
\hline 9 & Female (23) & 26 & MPAL & $12 \mathrm{~Gy} / 6 \mathrm{~F}$ b.i.d & $\mathrm{BM}$ \\
\hline 10 & Female (58) & 38 & Follicular lymphoma recurrence & $6 \mathrm{~Gy} / 2 \mathrm{~F} 1 \mathrm{~F} / \mathrm{d}$ & $\mathrm{BM}$ \\
\hline 11 & Male (27) & 29 & T-ALL CNS recurrence & $12 \mathrm{~Gy} / 6 \mathrm{~F}$ b.i.d & BM, CNS, Spleen, Scrotum \\
\hline 12 & Male (19) & 26 & Pre-B ALL recurrence & $12 \mathrm{~Gy} / 6 \mathrm{~F}$ b.i.d & BM, CNS, Spleen, Scrotum \\
\hline 13 & Male (20) & 22 & BPCDN & $12 \mathrm{~Gy} / 6 \mathrm{~F}$ b.i.d & $\mathrm{BM}$ \\
\hline 14 & Female (45) & 32 & Pre-B ALL & $12 \mathrm{~Gy} / 6 \mathrm{~F}$ b.i.d & BM, CNS, Spleen \\
\hline 15 & Male (21) & 23 & Pre-B ALL recurrence & $12 \mathrm{~Gy} / 6 \mathrm{~F}$ b.i.d & BM, CNS, Spleen, Scrotum \\
\hline 16 & Male (12) & 19 & Pre-B ALL recurrence & $11 \mathrm{~Gy} / 4 \mathrm{~F} 1 \mathrm{~F} / \mathrm{d}$ & BM, CNS, Spleen, Scrotum \\
\hline 17 & Male (30) & 28 & T-ALL & $12 \mathrm{~Gy} / 6 \mathrm{~F}$ b.i.d & BM, CNS, Spleen, Scrotum \\
\hline 18 & Male (30) & 25 & Pre-B ALL "Ph-like ALL" & $12 \mathrm{~Gy} / 6 \mathrm{~F}$ b.i.d & BM, CNS, Spleen, Scrotum \\
\hline 19 & Male (10) & 14 & Pre-B ALL Recurrence & $12 \mathrm{~Gy} / 6 \mathrm{~F}$ b.i.d & BM, CNS, Spleen, Scrotum \\
\hline 20 & Female (46) & 32 & Follicular lymphoma recurrence & $12 \mathrm{~Gy} / 6 \mathrm{~F}$ b.i.d & BM, CNS, Spleen \\
\hline \multirow[t]{2}{*}{21} & Male (29) & 28 & Pre-B ALL recurrence & $12 \mathrm{~Gy} / 6 \mathrm{~F}$ b.i.d & BM, CNS, Spleen, Scrotum \\
\hline & & & & Cranial boost $6 \mathrm{~Gy} / 3 \mathrm{~F}$ & \\
\hline 22 & Male (35) & 24 & Pre-B ALL & $12 \mathrm{~Gy} / 6 \mathrm{~F}$ b.i.d & BM, CNS, Spleen, Scrotum \\
\hline 23 & Female (42) & 26 & Myeloma & $12 \mathrm{~Gy} / 6 \mathrm{~F}$ b.i.d & $\mathrm{BM}$ \\
\hline
\end{tabular}




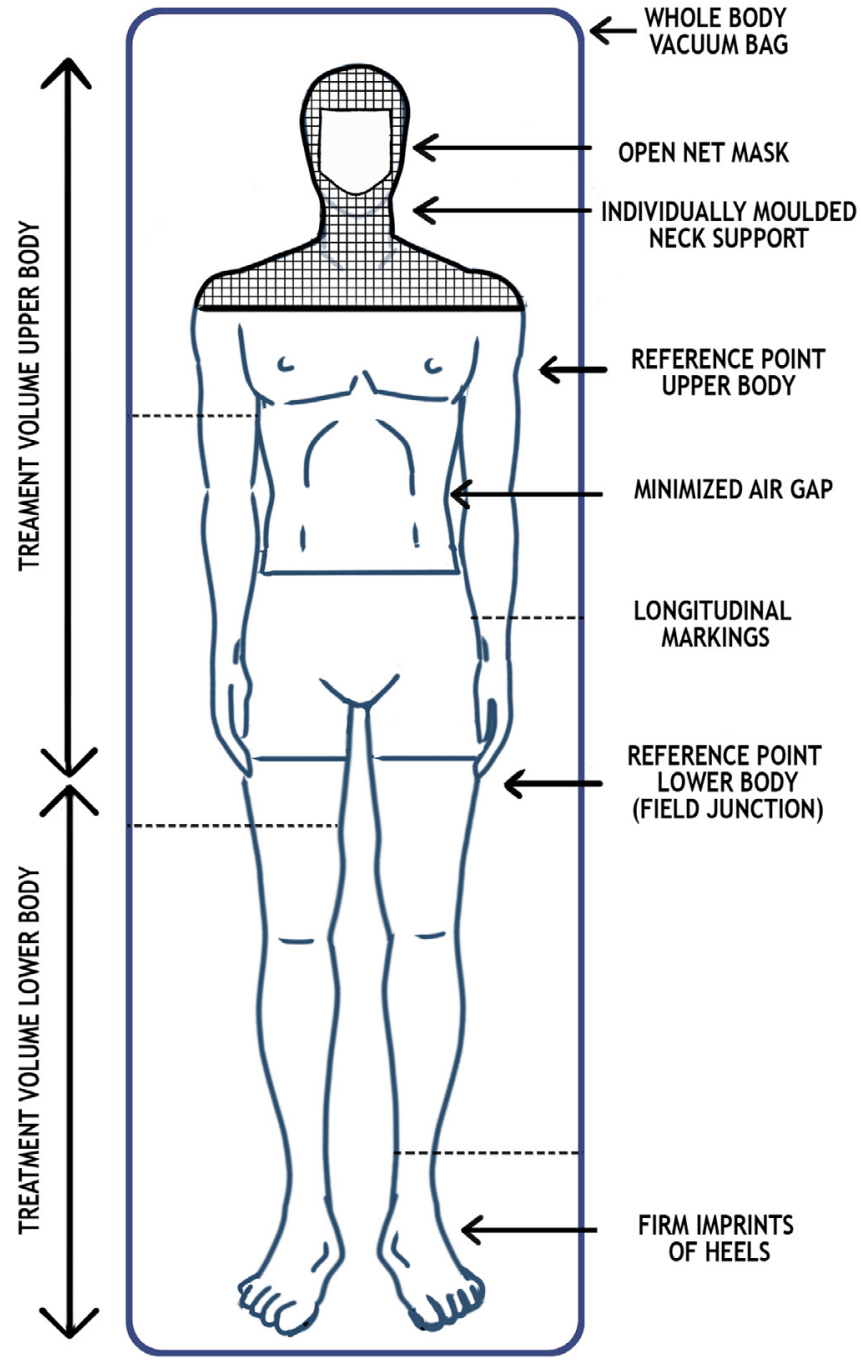

Fig. 1. Illustration of the patient setup including extension of treatment fields, immobilization equipment and reference points. Setup markings on patient are shown as dotted lines.

contoured by the TPS but manually corrected; ribs were joined to a single volume but cartilage was excluded ventrally. In addition, brain, spleen, lymph nodes and testes were included or omitted as target depending on the patient's diagnosis, as seen in Table 1. Structures considered to lack red bone marrow such as sesamoid bones, hyoid bone, larynx, patella, toes and the distal part of the fingers were excluded from CTV. Margins from CTV to PTV were applied based on established values used in our clinic for similar treatment sites, such as craniospinal irradiation (CSI) or total lymph node irradiation (TLI). For the torso an additional 3-mm margin was applied compared to $7 \mathrm{~mm}$ used for CSI and TLI, in all $10 \mathrm{~mm}$. For the head, immobilized with an open net mask, $2 \mathrm{~mm}$ was added to the $5 \mathrm{~mm}$ margin normally used for targets of the head and neck, in all $7 \mathrm{~mm}$. For patients having a left-to-right arm distance exceeding $40 \mathrm{~cm}$, a 13-15 mm PTV margin was generally used due to the $40 \mathrm{~cm}$ field of view (FOV) limitation of the MVCT.

To avoid optimization in air of superficial parts of the target (e.g. skull, clavicle and shins), optimization bolus was added extending $4 \mathrm{~mm}$ beyond the PTV with a density override of $0.6 \mathrm{~g} / \mathrm{cc}$. The technique and thickness of bolus used was similar to the one described by Moliner et al. [15], with the exception of the bolus density, where Moliner et al. used a density of $0.4 \mathrm{~g} / \mathrm{cc}$.

\subsection{Treatment planning}

After delineation, both CT scans along with corresponding structure sets were exported to the TomoTherapy TPS. Planning parameters were set according to clinical practice, initial testing and literature [16-19], as follows; a pitch of 0.397 , field width of $5 \mathrm{~cm}$, a modulation factor of 2.5-2.8 for the upper body. This pitch value was selected to minimise the thread effect for the especially lateral targets, such as the arms, and presents a good compromise between dose homogeneity, beam-on time and MLC segment complexity [18]. The same pitch and field width were used also for the lower body but the modulation factor was reduced to 1.6 to minimise treatment time. Planning optimization criteria were set to achieve $100 \%$ of the prescribed dose to cover $60 \%$ of the PTV and a minimum of $98 \%$ of prescribed dose to cover $95 \%$ of the PTV. Optimization was then continuously progressed until lowest possible doses to OARs were achieved with uncompromised PTV coverage.

Because of the lateral extension of the arms, the target coverage was generally lower than that of the torso and head. This can be mainly attributed to the threading effect [18], and due to the maximum field width of $40 \mathrm{~cm}$ at isocenter was less than the patient width. Multiple objectives were put on each OAR to minimise the dose. For each OAR, an optimization volume was created as a copy but separated $5 \mathrm{~mm}$ from the PTV. For lungs, a $10-\mathrm{mm}$ separation was used. This generally facilitates the optimization and reduces the risk of hotspots at the PTVOAR border.

The fields of the upper and lower body plans were matched at the upper thigh. This position presents the least complicated target geometry when merging two Tomotherapy fields since the target here only consists of the two femoral shafts with no nearby organs at risk. To create a robust plan with homogeneous dose across the field junction, shallow dose gradients were created in the optimisation as shown in Fig. 2. The target dose of the upper body was ramped down from $100 \%$ to $0 \%$ over the last $6 \mathrm{~cm}$ using six target segments of $1.0 \mathrm{~cm}$ assigned descending dose values of approximately $2 \mathrm{~Gy}$ per segment. The cranial dose of the lower body was ramped down correspondingly resulting in a homogeneous dose across the junction. This sizable dose ramping volume allows for a minor longitudinal mismatch of the two fields without compromising target coverage. After completing optimization, both dose matrices were exported to the Eclipse TPS for dose summation. The CT sets for the upper and the lower body were automatically registered based on anatomy, and manually corrected to align the fiducial thigh markers. In Eclipse (version 13.6), the correct image orientation of the registration is handled internally but is always visually confirmed afterwards.

To validate the choice of CTV-PTV margins and to verify that all patients received the prescribed dose, we applied the concept of Dose Coverage Histogram (DCH) [21]. Basically, the Dose Coverage Histogram tells the probability that the dose to the CTV is covered to a certain level based on the variation in DVHs calculated from a number of treatments. MVCT data were collected for all delivered fractions and the CTV structure position was corrected by rigid adjustment (translationally) slice by slice to match the target in treated position. The treatment plan was then recalculated based on the geometry at the time of treatment and compared to the original treatment plan. From these data, dose coverage histograms were calculated and evaluated. We evaluated the DCH for the 15 first patients.

To evaluate the dose homogeneity around the field junction from setup deviations, a recalculation was performed with an independent dose calculator. The upper and the lower body image set was moved $5 \mathrm{~mm}$ longitudinally in both directions, and $10 \mathrm{~mm}$ laterally and vertically in both directions. $5 \mathrm{~mm}$ longitudinal was used since the registration and setup during treatment was based on the longitudinal position of the junction markers. The recalculated datasets were combined in Python and evaluated based on the CTV $\pm 2.0 \mathrm{~cm}$ from the junction markers on the thigh. The robustness of the CTV dose homogeneity in the field junction was evaluated for 8 patients. 


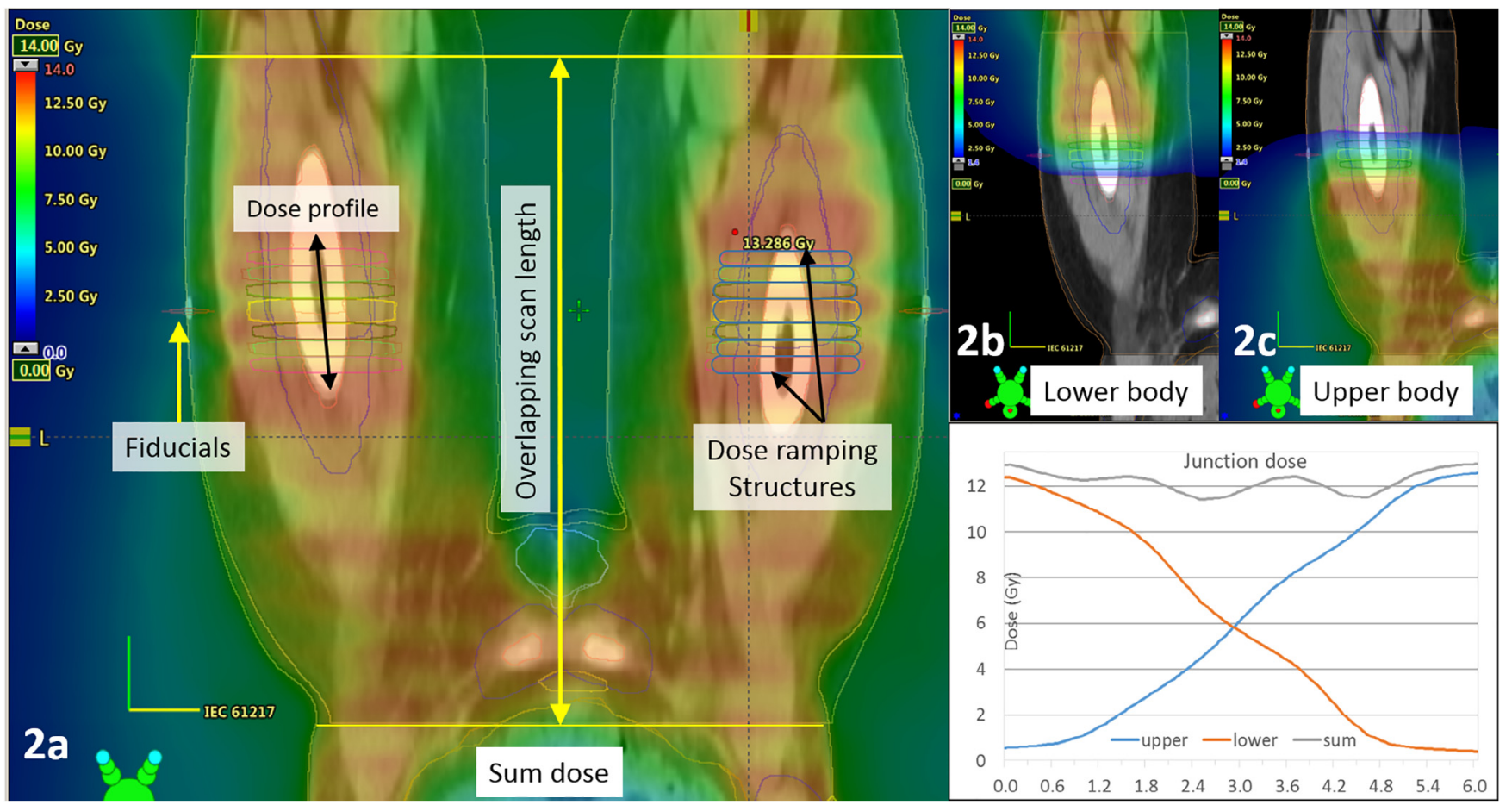

Fig. 2. Coronal plane of thigh area including junction section. Dose distribution from lower body plan and upper body plan (2b and 2c) add up to (2a). Lower right plot shows a 6-cm dose profile across the junction with individual plan contributions.

\subsection{Delivery quality control (DQC)}

A dose verification was performed on all patients before treatment start. For treatment DQC, the Delta4 phantom was used (ScandiDos, Uppsala, Sweden); a cylindrical PMMA phantom with a cross-plane diode array of 1069 diodes. Agreement between delivered and planned dose was evaluated with gamma analysis [20] in accordance with our clinical practice: $3 \%$ dose difference, $2 \mathrm{~mm}$ distance to agreement with a minimum pass rate of $90 \%$ with global dose normalization and a $15 \%$ threshold. Due to the extensive length of the target, multiple measurements were necessary to cover the entire volume.

\subsection{Imaging, registration \& treatment}

Patient positioning and imaging was performed with the aid of two independent systems: Sentinel optical surface scanning system (C-RAD, Uppsala, Sweden) (henceforth designated the surface guided radiotherapy (SGRT) system), and MVCT using the built-in detector array. Sentinel is a fast laser-based optical surface scanning system that acquires 3D images of the patient surface and calculates the position using rigid registration based on a reference scan or imported external structure from the TPS.

Before MVCT, radio-opaque fiducial markers were placed on the internal reference tattoos of the upper thigh, marking the position of the field junction. When treating the upper body, patients were positioned using the reference markings at mid-thorax level (Fig. 1). In case of left-to-right arm distance exceeding $40 \mathrm{~cm}$, the upper body was SGRT scanned prior to MVCT. After assuring proper positioning of the arms, an MVCT was acquired from vertex to mid-thigh. Image registration was performed automatically, reviewed, and if necessary adjusted manually. The longitudinal shift was also applied but not exceeding $5 \mathrm{~mm}$ from the junction markers in order to keep a homogeneous junction dose.

Prior to treatment of the lower body, the entire immobilization was rotated 180 degrees and the neck rest and mask were replaced with a pillow for patient comfort. Here, no longitudinal deviation in junction marker position was allowed at registration in order to maintain a homogeneous junction dose and only lateral or vertical corrections were applied if necessary.

In order to save patient time on the treatment couch, MVCT registration was replaced with SGRT for the lower body. Image registration data of MVCT and SGRT scans were taken sequentially for the first three fractions and analysed and correlated. If the two image sets correlated within $3 \mathrm{~mm}$, only SGRT was used for the lower body for the remaining fractions.

\subsection{Backup treatment preparation}

Being a single Tomotherapy unit centre, to prevent treatment course interruption in case of machine breakdown, a conventional TBI treatment plan was prepared for each patient as described by Svahn-Tapper et al. [21]. In vivo TLD or diode measurements performed at the first fractions were used for dose verification and if necessary also a dose correction. A conventional linac was kept in stand-by to complete the TMI treatment with the TBI backup treatment if required. In case of interruption during treatment, the partial TMI treatment length was calculated based on elapsed treatment time and couch travel parameters, and the treatment field of the backup TBI plan was matched at the skin where the Tomotherapy treatment ended. This procedure does not aim at perfect matching of the field junction but rather to avoid a larger underdosage. Instead, an overdosage of a small section is anticipated and each case is reviewed by the radiation oncologist.

\section{Results}

Since October 2014, twenty-three patients have been treated with TMI (Table 1). All patients completed their treatment as planned and could proceed with subsequent SCT without delay. Two patients received partial TBI backup treatment due to temporary machine malfunction. None of the patients required anaesthesia, but three patients received mild sedatives during some of the TMI fractions due to anxiety. Dose to targets are reported as near minimum and near maximum dose 
Table 2

Mean dose $\left(D_{\text {mean }}\right)$ to organs at risk, near maximum dose $\left(D_{2 \%}\right)$ and near minimum $\left(\mathrm{D}_{98 \%}\right)$ dose to target with lower body and upper body plans summarized but excluding any boost treatment. Doses are normalized to the prescribed dose and patients are numbered consecutively. The transition volume (PTV Trans ), extends $2 \mathrm{~cm}$ cranio-caudally across the junction.

\begin{tabular}{|c|c|c|c|c|}
\hline & Mean (\%) & SD (\%) & Range (\%) & \\
\hline $\mathrm{PTV}_{\text {Thorax }} \mathrm{D}_{98 \%}$ & 94.5 & 2.1 & 87.1 & 98.4 \\
\hline PTV $_{\text {Thorax }} \mathrm{D}_{2 \%}$ & 106.6 & 1.2 & 104.9 & 110.6 \\
\hline $\mathrm{PTV}_{\text {Thorax }} \mathrm{D}_{\text {mean\% }}$ & 100.6 & 1.1 & 96.2 & 101.9 \\
\hline PTV $_{\text {Legs }} \mathrm{D}_{98 \%}$ & 95.6 & 3.5 & 89.6 & 101.1 \\
\hline PTV $_{\text {Legs }} \mathrm{D}_{2 \%}$ & 109.0 & 3.8 & 101.5 & 118.3 \\
\hline PTV $_{\text {Legs }} \mathrm{D}_{\text {mean }}$ & 102.6 & 2.3 & 95.8 & 107.5 \\
\hline PTV $_{\text {trans }} \mathrm{D}_{98 \%}$ & 94.8 & 4.1 & 88.7 & 104.0 \\
\hline $\mathrm{PTV}_{\text {Trans }} \mathrm{D}_{2 \%}$ & 109.6 & 5.2 & 101.9 & 122.0 \\
\hline Lungs $\mathrm{D}_{\text {mean }}$ & 77.3 & 3.3 & 70.1 & 87.5 \\
\hline Liver $D_{\text {mean }}$ & 69.0 & 5.2 & 59.7 & 79.2 \\
\hline Heart $D_{\text {mean }}$ & 61.1 & 5.7 & 53.0 & 75.1 \\
\hline Kidneys $\mathrm{D}_{\text {mean }}$ & 62.3 & 5.2 & 54.7 & 77.7 \\
\hline Bowelbag $\mathrm{D}_{\text {mean }}$ & 61.3 & 4.2 & 52.8 & 71.0 \\
\hline
\end{tabular}

whereas OARs are reported as average doses (Table 2). All doses to OARs are below those previously received by patients treated with standard TBI. Dose calculated in the Eclipse TPS simulating a TBI treatment geometry yields an average dose of $99 \%$ of the prescribed target dose to kidneys, heart, bowel bag and liver for a patient of average BMI. The average dose to kidneys, heart and bowel was reduced by approximately $40 \%$ with the TMI treatment compared to the conventional TBI treatment. Typically, one day was allocated for sessions of immobilization and CT, one day to TBI immobilization and planning for backup purpose, one day to delineation, and 3-4 days to planning, DQC and treatment preparation.

\subsection{Planning}

The values of planning parameters and optimization objectives differed only little between patients. For the upper body, the median values of modulation factor and beam-on time were $1.94 \pm 0.13$ and $21.0 \pm 2.0 \mathrm{~min}$ respectively and corresponding values for the lower body were 1.3 and $9 \mathrm{~min}$. Except for the first patient, all patients receiving $2.0 \mathrm{~Gy} /$ fraction were planned with a field width of $5 \mathrm{~cm}$ and pitch value of 0.397 . Hence a class solution was created and used for planning almost all of the patients, cutting planning time considerably. Fig. 2 shows a coronal plane of the junction volume at and the dose distribution from each plan summed up to the total dose.

For all patients calculated with DCH, the probability of achieving dose coverage of at least $98 \%$ of prescribed dose to at least $93 \%$ of the target volume was on average $\mathrm{P}\left[\mathrm{D}_{98 \%} \geq 93 \%\right]=0.9(0.8-1.0)$ for delivered fractions, and 1.0 for all planned fractions.

All treatment plans passed the QC measurements using the Delta4 detector, with an average gamma pass rate of $97 \%$ (91-100\%).

\subsection{Image registration and treatment}

Optical surface scanning using the Sentinel system was initially tested on both upper and lower body to replace MVCT and save patient time on the treatment couch. A time analysis of the radiation treatment procedure was performed where 11 different steps were timed and noted in order to identify procedures that could be streamlined. The treatment time (patient entrance to exit) was on average $1 \mathrm{~h} 56 \mathrm{~min}$ $(1: 44-2: 07 \mathrm{~h})$ for treatment fractions with MVCT on both lower and upper body, and on average $1 \mathrm{~h} 31 \mathrm{~min}(1: 25-1: 36 \mathrm{~h})$ for treatment fractions with MVCT on upper body and surface scanning positioning on lower body. The difference in setup time was significant, as tested with Mann-Whitney two-sided $U$ test $(\mathrm{p}<0.005)$ indicating that the SGRT system (Median $=12 \mathrm{~min}$ ) is faster for setup of lower body than
MVCT (Median $=31 \mathrm{~min}$ ).

For the lower body, the 3-mm correlation criteria were met for most but not all patients. For one patient that showed exceptional setup reproducibility after the first three fractions, SGRT was also performed on the upper body and reduced the total treatment time (entrance to exit) to $60 \mathrm{~min}$. For the rest of the patients, the flexibility of joints and bony structures resulted in too large daily variations and IGRT was restricted to the lower body only. However, SGRT continued to be used for registering the position of the arms due to the insufficient FOV of the Tomotherapy MVCT.

The offset plans had a mean $\mathrm{D}_{95 \%}=1.85 \mathrm{~Gy}(\mathrm{SD}=0.11 \mathrm{~Gy})$ and a mean $\mathrm{D}_{5 \%}=2.24 \mathrm{~Gy}(\mathrm{SD}=0.15 \mathrm{~Gy})$ with the original plans had a $\mathrm{D}_{95 \%}=1.89 \mathrm{~Gy} \quad(\mathrm{SD}=0.04 \mathrm{~Gy}) \quad$ and $\quad \mathrm{a}$ mean $\mathrm{D}_{5 \%}=2.21 \mathrm{~Gy}$ $(\mathrm{SD}=0.06 \mathrm{~Gy})$.

\section{Discussion}

Radiotherapy plays an important part of the conditioning prior to bone marrow transplantation. The traditional technique, TBI with shielded lungs, exhibit large dose heterogeneity and high doses to OARs. The technique implemented in this work, TMI with HT, significantly reduces the doses to OARs, but is more demanding on optimization of treatment, immobilization, quality assurance, treatment planning and beam delivery. To our knowledge, this is the first published method to incorporate backup planning and SGRT in TMI treatment. The fact that two patients were transferred to a conventional linac for partial TBI treatment completion confirms the need for a backup solution in case of e.g. machine failure or other unexpected circumstances.

The immobilization technique used was based on extensive experience with HT from similar treatments of long targets, such as craniospinal irradiation and total lymph node irradiation. Our strategy for a safe treatment was to define PTVs with margins sufficiently large to avoid repositioning with a lengthy rescanning and registration, while still managing significant OAR sparing. Compared to most other studies, we have initially opted for more generous PTV margins to minimise the risk of missing targets and hence report slightly higher doses to sensitive organs than other publications [22]. Movement of the ribs from free breathing poses no problem since a normal 3-4 $\mathrm{mm}$ anteriorposterior amplitude is covered by the $10-\mathrm{mm}$ PTV. The applied margins have shown to be appropriate based on reviewed images and dose coverage histograms, but there may be room for reduction in certain areas of the body. A larger patient cohort may identify where the setup variation is at its largest and smallest. Risk organs of smaller children are especially difficult to spare because of the large PTV volume relative to the body but can benefit significantly from a mere 2-mm PTV shrinkage. Nevertheless, even among the paediatric patients, considerable sparing of OARs were obtained with the present margins. In this study we focussed on reducing dose to a smaller number of organs. Retrospective data of from TBI treatments show that the late side effects mainly concern kidneys, lungs and liver but also bowel bag and heart [23]. By forcing to reduce dose to numerous sensitive organs, an increased dose heterogeneity is introduced and hence an increased complexity of the treatment. This may convey risks and needs to be carefully thought through.

Another advantage with the TMI treatment is that optimisation in the Tomotherapy TPS gives the radiation oncologist full control over customizing target extensions and dose differentiation. For example, Patient \#6 received an additional boost to $20 \mathrm{~Gy} / 5 \mathrm{~F}$ to the right humerus and thoracic columna due to FDG-PET positive myeloma involvement, and patient \#10 received a boost to enlarged lymph nodes.

In contrast to a few other published studies, we have opted for the helical technique throughout the treatment as opposed to the mixed technique that adjoins the helical irradiation field of the upper body with anterior-posterior fields on the lower body using a conventional accelerator [24]. Reasons for this are a better control of the dose to the 
junction area, a superior dose distribution and avoiding transit between treatment units. We have consistently planned with the fixed jaw option by tradition, but dynamic jaw works equally well.

This work describes an effortless procedure to plan, match and add the doses from the upper and lower body plans without DICOM file manipulation as described by Zeverino et al. [14]. By introducing an optimised shallow gradient of the abutting fields at the junction, the robustness is greatly improved. A very similar approach has been described by Mancosu et al in their TMI approach using VMAT [25].

The time required for delineation can possibly be reduced by autocontouring, such as atlas-based segmentation. Planning time was considerably reduced by using a class solution for TMI optimization and an acceptable plan is generally achieved within a day. A combination of gained experience and the use of SGRT has cut treatment time substantially and is today comparable to TBI treatment time slots. SGRT has shown great potential to reduce time on the treatment table for the patient but needs to be further investigated and developed into e.g. a multi-camera system. Such a system could also be used to monitor patient motion in real time by compensating the couch motion and quantify the intra-fraction motion in a way that is not yet available for Tomotherapy treatments. This would improve treatment safety especially for patients with very long treatment times such as TMI. However, the Sentinel system may still be used to confirm that the patient position is still unchanged, by acquiring a second surface scan immediately after the treatment to compare with the pre-treatment scan.

In addition, from our robustness analysis of the junction volume we can conclude that the field matching technique is safe within normal setup deviations.

Compared to TBI, TMI with HT is able to deliver a more uniform dose to bone marrow and significantly reduce dose to OAR and allow for sophisticated individual dose delivery including simultaneously treating boost volumes. After 23 treated patients, no increased toxicity, recurrence or severity of GVHD have been reported as compared to the conventional TBI technique.

\section{Conclusion}

In this paper, we have described a procedure of implementing TMI in a clinical routine using a multidisciplinary team with members of each involved staff group. By forming a dedicated TMI team already from the beginning, procedures were consistently followed and continuously improved. Critical steps in the process have been identified and refined using various robustness techniques. TMI protocol has resulted in very reproducible treatments regardless of anatomy or conditioning of the patients. Feedback and new ideas are still discussed at regular TMI group meetings and has been a vital part of the implementation procedure.

\section{Funding details}

Department of corresponding author have an ongoing research agreement with Accuray Inc. which includes funding.

\section{Ethics}

Approved by local ethics committee, written consent from patients and all patient information have been fully anonymised.

\section{References}

[1] Adkins DR, DiPersio JF, Adkins DR. Total body irradiation before an allogeneic stem cell transplantation: Is there a magic dose? Curr Opin Hematol 2008;15:555-60. https://doi.org/10.1097/MOH.0b013e32831188f5.

[2] Hartman A-R, Williams SF, Dillon JJ. Survival, disease-free survival and adverse effects of conditioning for allogeneic bone marrow transplantation with busulfan/ cyclophosphamide vs total body irradiation: a meta-analysis. Bone Marrow Transplant 1998;22:439-43. https://doi.org/10.1038/sj.bmt.1701334.

[3] Giralt SA, LeMaistre CF, Vriesendorp HM, Andersson BS, Dimopoulos M, Gajewski J, et al. Etoposide, cyclophosphamide, total-body irradiation, and allogeneic bone marrow transplantation for hematologic malignancies. J Clin Oncol 1994;12:1923-30. https://doi.org/10.1200/JCO.1994.12.9.1923.

[4] Clift RA, Buckner CD, Appelbaum FR, Bryant E, Bearman SI, Petersen FB, et al. Allogeneic marrow transplantation in patients with chronic myeloid leukemia in the chronic phase: a randomized trial of two irradiation regimens. Blood 1991;8:1660-5.

[5] Peñagarícano JA, Chao M, Rhee FV, Moros EG, Corry PM, Ratanatharathorn V. Clinical feasibility of TBI with helical tomotherapy. Bone Marrow Transplant 2011;46:929-35. https://doi.org/10.1038/bmt.2010.237.

[6] Nalichowski A, Eagle DG, Burmeister J. Dosimetric evaluation of total marrow irradiation using 2 different planning systems. Med Dosim 2016;41:230-5. https:// doi.org/10.1016/j.meddos.2016.06.001.

[7] Schultheiss TE, Wong J, Liu A, Olivera G, Somlo G. Image-guided total marrow and total lymphatic irradiation using helical tomotherapy. Int J Radiat Oncol Biol Phys 2007;67:1259-67. https://doi.org/10.1016/j.ijrobp.2006.10.047.

[8] Corvò R, Zeverino M, Vagge S, Agostinelli S, Barra S, Taccini G, et al. Helical tomotherapy targeting total bone marrow after total body irradiation for patients with relapsed acute leukemia undergoing an allogeneic stem cell transplant. Radiother Oncol 2011;98:382-6. https://doi.org/10.1016/j.radonc.2011.01.016.

[9] Somlo G, Spielberger R, Frankel P, Karanes C, Krishnan A, Parker P, et al. Total marrow irradiation: a new ablative regimen as part of tandem autologous stem cell transplantation for patients with multiple myeloma. Clin Cancer Res 2011;17:174-82. https://doi.org/10.1158/1078-0432.CCR-10-1912.

[10] Gruen A, Ebell W, Wlodarczyk W, Neumann O, Kuehl JS, Stromberger C, et al. Tota body Irradiation (TBI) using Helical Tomotherapy in children and young adults undergoing stem cell transplantation. Radiat Oncol 2013;8:92. https://doi.org/10. 1186/1748-717X-8-92.

[11] Wong JYC, Forman S, Somlo G, Rosenthal J, Liu A, Schultheiss T, et al. dose escalation of total marrow irradiation with concurrent chemotherapy in patients with advanced acute leukemia undergoing allogeneic hematopoietic cell transplantation. Int J Radiat Oncol Biol Phys 2013;85:148-56. https://doi.org/10.1016/j.ijrobp. 2012.03 .033

[12] Hui SK, Verneris MR, Higgins P, Gerbi B, Weigel B, Baker SK, et al. Helical tomotherapy targeting total bone marrow first clinical experience at the University of Minnesota. Acta Oncol 2007;46:250-325. https://doi.org/10.1080/ 02841860601042449 .

[13] Zhuang AH, An L, Schultheiss TE, Wong JYC. Dosimetric study and verification of total body irradiation using helical tomotherapy and its comparison to extended SSD technique. Med Dosim 2010;35:243-9. https://doi.org/10.1016/j.meddos. 2009.07.001.

[14] Zeverino M, Agostinelli S, Taccini G, Cavagnetto F, Garelli S, Gusinu M, et al. Advances in the implementation of helical tomotherapy-based total marrow irradiation with a novel field junction technique. Med Dosim 2012;37:314-20. https:// doi.org/10.1016/j.meddos.2011.12.001.

[15] Moliner G, Izar F, Ferrand R, Bardies M, Ken S, Simon L. Virtual bolus for total body irradiation treated with helical tomotherapy. J Appl Clin Med Phys 2015;16:164-76. https://doi.org/10.1120/jacmp.v16i6.5580.

[16] Kissick MW, Fenwick J, James JA, Jeraj R, Kapatoes JM, Keller H, et al. The helical tomotherapy thread effect. Med Phys 2005;32:1414-23. https://doi.org/10.1118/ 1.1896453.

[17] Chen M, Chen Y, Chen Q, Lu W. Theoretical analysis of the thread effect in helical TomoTherapy. Med Phys 2011;38:5945-60. https://doi.org/10.1118/1.3644842.

[18] Takahashi Y, Verneris M, Dusenbery K, Wilke C, Weisdorf DJ, Hui SK. Peripheral dose heterogeneity due to the thread effect in total marrow irradiation with helical tomotherapy. Int J Radiat Oncol Biol Phys 2013;87:832-9. https://doi.org/10. 1016/j.ijrobp.2013.07.017.

[19] Haraldsson A, Hauer Karlsson A, Ambolt L, Engström P. Plan specific pitch on Tomotherapy-plans effect on gamma pass rate for patient QA measured on Delta4. Radiother Oncol 2016;119:S740. https://doi.org/10.1016/S0167-8140(16) 32843-2.

[20] Low DA, Dempsey JF. Evaluation of the gamma dose distribution comparison method. Med Phys 2003;30:2455-64. https://doi.org/10.1118/1.1598711.

[21] Svahn-Tapper G, Nilsson P, Jönsson C, Alvegård TA. Calculation and measurements of absorbed dose in total body irradiation. Acta Oncol 1990;29:627-33. https://doi org/10.3109/02841869009090064.

[22] Paix A, Antoni D, Waissi W, Ledoux MP, Bilger K, Fornecker L, et al. Total body irradiation in allogeneic bone marrow transplantation conditioning regimens: a review. Crit Rev Oncol Hematol 2018;123:138-48. https://doi.org/10.1016/j. critrevonc.2018.01.011

[23] Leiper AD. Late effects of total body irradiation. Arch Dis Child 1995;72:382-5.

[24] Wong JYC, Liu A, Schultheiss T, Popplewell L, Stein A, Rosenthal J, et al. Targeted total marrow irradiation using three-dimensional image-guided tomographic intensity-modulated radiation therapy: an alternative to standard total body irradiation. Biol Blood Marrow Transplant 2006;12:306-15. https://doi.org/10.1016/j bbmt.2005.10.026.

[25] Mancosu P, Navarria P, Castagna L, Reggiori G, Stravato A, Gaudino A, et al. Plan robustness in field junction region from arcs with different patient orientation in total marrow irradiation with VMAT. Phys Med 2015;31:677-82. https://doi.org/ 10.1016/j.ejmp.2015.05.012. 